\title{
Development the Measurement System of the Multi-Channel Frequency Standard Comparison
}

\author{
Zhang Yue ${ }^{1,}$ *, Liu Yanqi ${ }^{2}$, Lin Tao ${ }^{1}$, Yuan Xiaodi ${ }^{1}$, Zhang Ammin ${ }^{1}$, Wang Yuzhuo ${ }^{1}$ \\ ${ }^{1}$ National Institute of Metrology, Beijing, China \\ ${ }^{2}$ Xinghan Technology Inc, Beijing, China
}

Email address:

zhangyue@nim.ac.cn (Zhang Yue)

${ }^{*}$ Corresponding author

\section{To cite this article:}

Zhang Yue, Liu Yanqi, Lin Tao, Yuan Xiaodi, Zhang Ammin, Wang Yuzhuo. Development the Measurement System of the Multi-Channel Frequency Standard Comparison. Journal of Electrical and Electronic Engineering. Vol. 9, No. 4, 2021, pp. 116-122. doi: $10.11648 /$ j.jeee.20210904.13

Received: July 14, 2021; Accepted: July 29, 2021; Published: August 4, 2021

\begin{abstract}
This paper introduces a measurement system and method of high-speed sampling and full digital for the comparison of multi-channel frequency standard. The hardware of the system consists of 4 parts: A: Impedance matching module (After matching with the impedance of the acquisition circuit, the two input signals are transformed into suitable amplitude, and then transformed into digital signals by the high-speed acquisition circuit). B: High speed data acquisition module. C: Data real-time processing module. D: Calculation module for phase difference and frequency stability. The system not only can achieve real-time processing under the minimum computing resources, but also provide the upper computer with complete information and equivalent frequency data with small amount of data. Moreover, the fast and high-precision calculation of phase difference and frequency stability can be achieved by improving the calculation process. The software of the system meets the requirements for the technical specifications of national metrology. Compared with domestic and foreign frequency standard comparators, the interface of software is more friendly, the operation is more convenient, and it has a strong ability to analyze and process data. The test results show that the uncertainty of the system is better than $1.5 \times 10^{-13}$ (sampling time is 1 second), which is consistent with Russian VCH-315 index.
\end{abstract}

Keywords: Frequency Standard Metrology, Frequency Standard Comparison, Frequency Stability, Dual Mixer Time Difference Measurement (DMTD)

\section{Introduction}

Frequency standard comparator is a measurement instrument for the parameter of time and frequency, widely used in frequency measurement in time and frequency field. The time difference method, phase comparison method, frequency difference multiplication method and dual mixer time difference method are the main precision measurement techniques in the field of time and frequency. Due to the different principles for realization, the comparison accuracy is not the same. At present, the dual mixer time difference method is a mainstream technique to achieve high precision frequency standard comparison [1, 2]. Most of the international equipment for frequency standard comparison adopt this technology to measure the frequency standard characteristics [3], mainly including American Microchip's $5120 \mathrm{~A}$ or $3120 \mathrm{~A}$ and Russia's VCH-314 or VCH-315, etc., with world-leading indexes, as shown in Table 1, in which the background noise of 5120A has reached the level of $10^{-15} / 1 \mathrm{~s}$ [4-6]. The background noise of $\mathrm{VCH}-314$ is also better than that of $2 \times 10^{-14} / 1 \mathrm{~s}$ (the measured results reach the level of $\left.10^{-15}\right)$, and the background noise of $\mathrm{VCH}-315$ (8 channel) is better than that of $1.5 \times 10-13 / 1 \mathrm{~s}$.

Table 1. Comparison for foreign mainstream comparator of frequency standard.

\begin{tabular}{llll}
\hline Type/Sample Time & $\mathbf{5 1 2 0 A}$ & VCH-314 & VCH-315 (8 channel) \\
\hline $1 \mathrm{~s}$ & $<3 \times 10^{-15}$ & $<2.0 \times 10^{-14}$ & $<1.5 \times 10^{-13}$ \\
$10 \mathrm{~s}$ & -- & $<5.0 \times 10^{-15}$ & $<2.0 \times 10^{-14}$ \\
$100 \mathrm{~s}$ & -- & $<1.5 \times 10^{-15}$ & $<3.0 \times 10^{-15}$ \\
$1000 \mathrm{~s}$ & -- & $<5.0 \times 10^{-16}$ & $<5.0 \times 10^{-16}$ \\
\hline
\end{tabular}


In China, the measurement resolution of the traditional dual mixer time difference DMTD equipment is mainly related to the measurement error of the time interval and the zero-crossing trigger error [7]. In fact, in recent years, Shijiazhuang Shuying, Chengdu Tianao and Xi 'an Hongtai have also made great progress in the technical research and production. The comparison uncertainty can reach between $10^{-12} \sim 10^{-13} / 1 \mathrm{~s}$.

With the continuous development of digital technology and the continuous improvement for hardware performance of digital signal processing, the measurement accuracy has been significantly improved compared with the traditional method. The technical indexes realized in this paper are consistent with the Russian VCH-315 (comparison uncertainty is better than $1.5 \times 10^{-13} / \mathrm{s}$ ) index. With no gap sampling and multiple selection of sampling times [8], it has realized the measurement of 8 channel for frequency signal $(5 / 10 \mathrm{MHz})$ and phase difference, relative frequency deviation, frequency stability, frequency daily fluctuation, frequency aging rate and frequency drift rate can be measured at the same time. It has reached the leading level in China and is also at advanced level in the world.

\section{Basic Principle of Digital Dual Mixer Time Difference Measurement}

The basic principle of the measurement system for the digital dual mixer time difference is shown in Figure 1 [2]. After the measured frequency standard signal (DUT) (device under test) and the reference (REF) frequency standard signal pass through the power divider respectively, the signal is divided into four channels and sampled by the analog-to-digital converter (ADC). The sampled signal of each channel is processed by the digital phase discriminator module (DPD) to get the phase data, and the phase difference data is obtained by subtraction of the phase data of the measured signal and the reference signal. Among them, four ADC modules and four DPD modules are synchronized by a common clock. It can be seen from the figure that the measurement system is two parts of upper and lower symmetry, and the output results are all the phase difference data of the measured signal and the reference signal. Then the cross-correlation operation is performed on the phase difference data of the two channels (the calculation of cross-variance), and the Allan deviation is finally obtained.

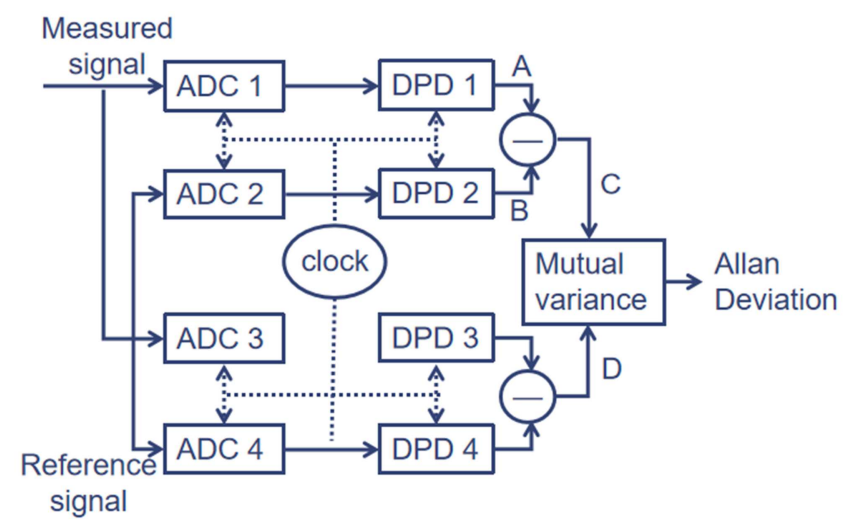

Figure 1. Principle of the measurement system for digital dual mixer time difference.

\section{The Hardware Composition of Multi-channel Frequency Standard Comparision Measurement System}

The hardware of the multi-channel frequency standard comparison measurement system is composed of four modules, namely: impedance matching module, signal acquisition module, real-time data processing module and phase difference and frequency stability calculation module. The overall design is shown in Figure 2.

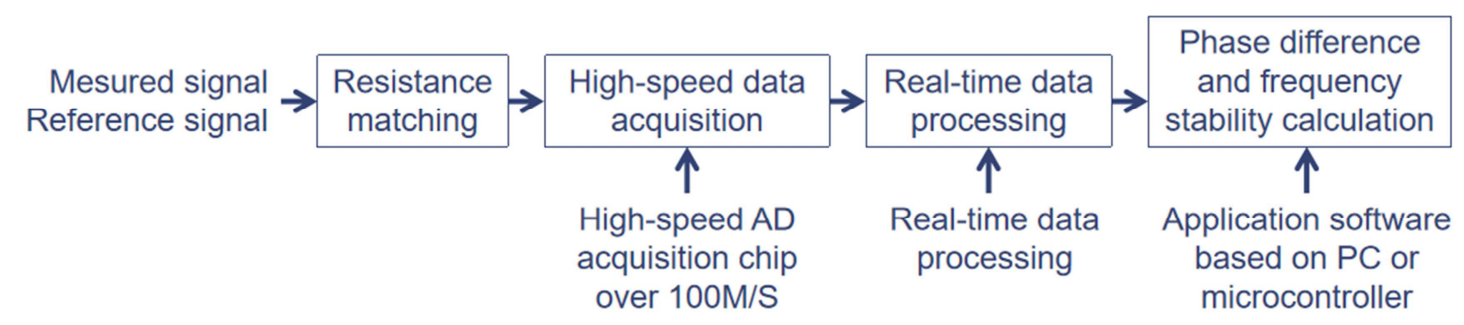

Figure 2. Design diagram of hardware.

The measured signal and reference signal are converted into acquisition signal with appropriate amplitude through impedance matching module, and then converted into digital signal by high-speed acquisition circuit. Then the real-time data processing module carries out parallel processing of a two-channel data on FPGA or multi-core DSP. Due to the high sampling frequency, which is generally in the order of $100 \mathrm{M} / \mathrm{S}$, the sampling data containing the measurement information must be processed in real time into a data stream with a lower bit rate. Only in this way can the phase difference and frequency stability calculation module be used for subsequent calculation on the ordinary PC or other single-chip computer systems.

\subsection{High-speed Data Acquisition and Real-time Data Processing}

The flow of high-speed data acquisition and real-time data processing is shown in Figure 3. After sampling, the reference signal FR and the measured signal FM (its standard frequency is $\mathrm{F} 0)$ are mixed with the sinusoidal data $\mathrm{FH}(\mathrm{FH}$ frequency is 
F0-FD, FD is the carrier frequency of difference frequency, which is generally far less than F0) generated inside the data processing chip respectively, that is, the data is multiplicated. Then the difference frequency signal is obtained through the low pass filter LP1, and its standard frequency is FD. The processing of reference signal and measured signal is a parallel process. In order to reduce the amount of data sent to the upper computer, multi-level filtering and extraction of data are carried out to reduce the signal sampling frequency (over hundreds of megabytes/second) to the acceptable range (generally below 1 megabytes/second) for subsequent CPU processing. In order to prevent the signal from containing other components and causing sampling aliasing, low-pass filtering should be carried out on the signal before each step of sampling. The filter is LP2. Finally, the data stream with low sampling bit rate is sent to PC or other CPU upper computer for subsequent processing.

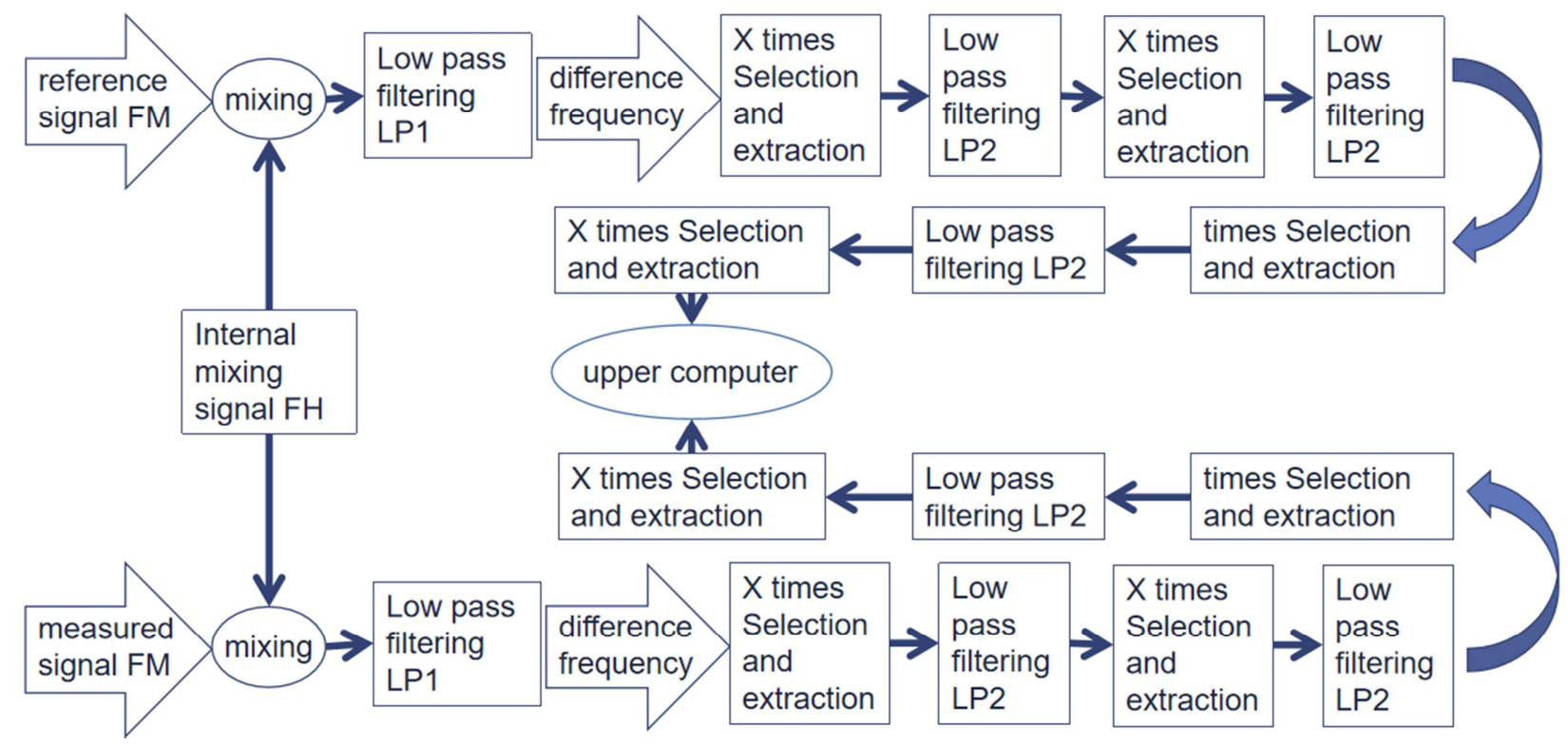

Figure 3. Flow chart of High-speed sampling data and data processing for real time.

\subsection{Phase Difference and Frequency Stability Calculation Procession}

The low bit rate data after real-time processing is sinusoidal data, and the phase difference of the two data channels can only be calculated by centralized processing of the whole data. The processing flow of phase difference and stability calculation is shown in Figure 4. Data of two channels are mixed with data (digital mixing signal) internally generated by CPU respectively, that is, the data is multiplied, and the low-frequency component near frequency 0 is retained by low-pass filtering. Then, the phase of each data channel is calculated by using the method of positive and inverse strings to obtain the phase data (range: $-Л \sim \pi$ ). In order to facilitate the calculation of phase difference (that is, the linear tilt component in the phase is recovered), the phase data should be restored and unwrapped, as shown in Figure 5. The linear tilt component is obtained by subtracting the linear tilt component in the phase of the two signals, that is, the phase difference.

In order to further filter out the noise in the phase difference data, low-pass filtering can be performed on the initial phase data after two-channel mixing. Since the linear tilt component of the phase corresponds to a fixed frequency difference, this part of data has no impact on the calculation of frequency stability, so the linear tilt component should be removed before filtering to obtain the residual phase data. Such processing is conducive to reducing noise and maintaining the integrity of the phase data. Before filtering, $\mathrm{N}$ times selection of residual phase data can reduce the data sampling frequency, reduce the order of the filter and reduce the computation. In order to adapt to different test objects, the filter here is designed as optional bandwidth filter. For the measured signal with poor frequency stability and large noise, the low-pass filter with higher bandwidth can be selected. For the measured signal with higher frequency stability and less noise, the low-pass filter with lower bandwidth can be selected. After filtering, the phase data is calculated and the residual phase difference is obtained by subtracting the phase data of the two channels.

The complete phase difference data of the two channels can be calculated by summing the phase difference of the linearly inclined component and the residual phase difference of the filter. The relative frequency deviation can be obtained by dividing the complete phase difference by time, and then the frequency stability of the two channels can be calculated.

\section{Data Processing Software}

The software is responsible for managing the data processing of the multi-channel frequency standard comparison test system and generating the calculation results of the frequency characteristics required by the national metrology technical specifications. [9-15] The software uses Visual $\mathrm{C}++$ programming language. Figure 6 is the software 
schematic diagram of the multi-channel frequency standard comparison test system. The software in the form of data, curves, charts, etc. can display the measurement results intuitively. It is easy to operate and it has intuitive interface.

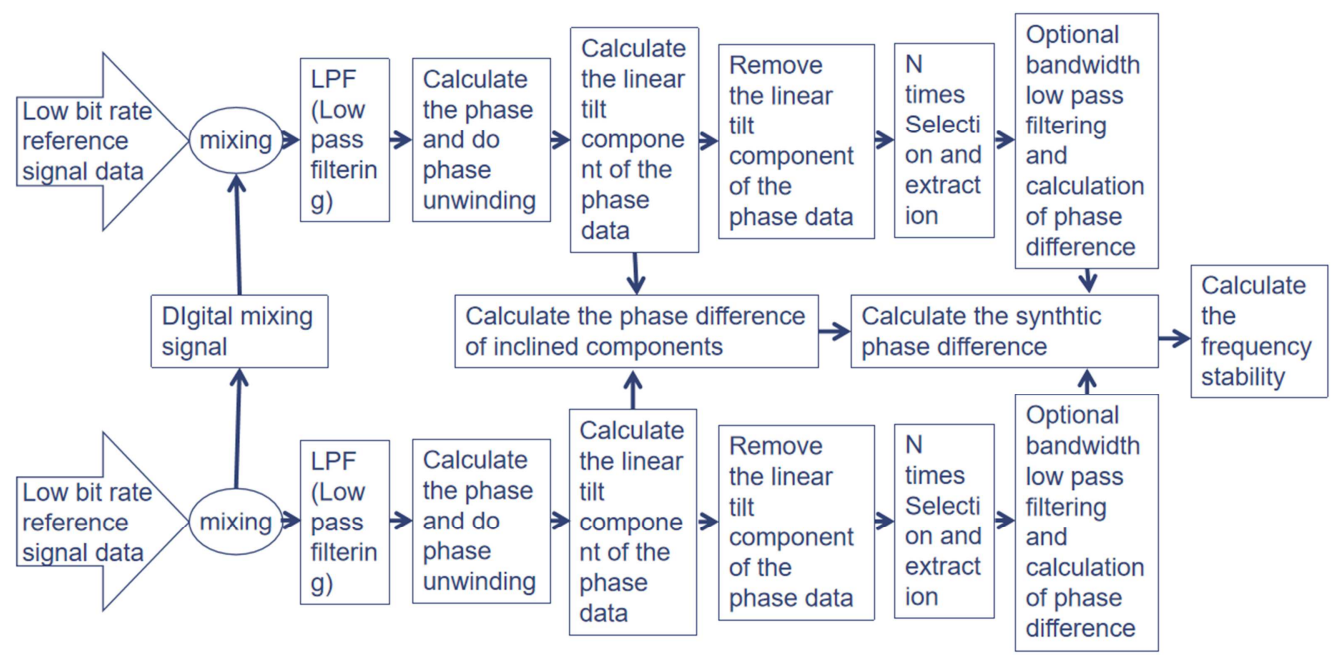

Figure 4. Processing flow chart of phase difference and calculation of frequency stability.
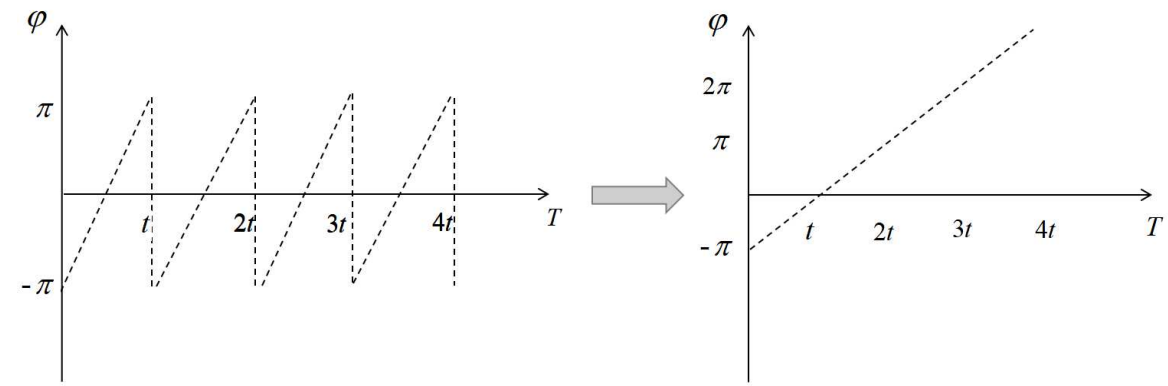

Figure 5. Principle of phase unwrapping.

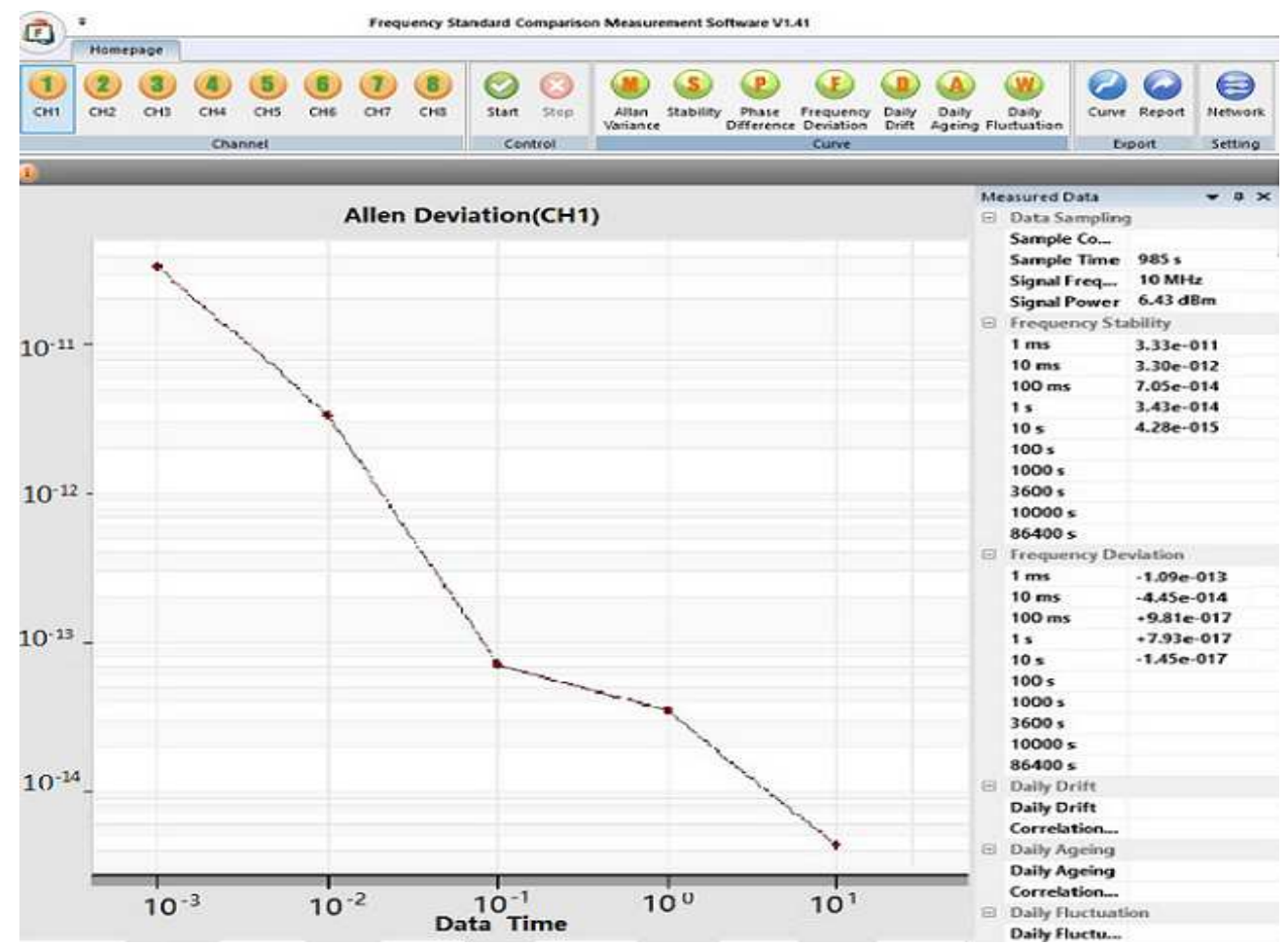

Figure 6. Software diagram of system. 


\section{Measurement Experiment of Multi-channel Frequency Standard Comparison System}

To verify the performance of multi-channel frequency standard comparison system, design two kinds of test schemes: background measurement experiment and comparative measurement experiment. Background measurement experiment is used to analyze the background noise of multi-channel frequency standard comparison system. The comparative measurement experiment is used to compare the comparative measurement results between different phase noise measurement systems.

\subsection{Experimental Results of Background Noise Measurement}

The cesium frequency standard $10 \mathrm{MHz}$ signal output end is connected to the frequency distribution amplifier, and the distribution amplifier outputs 9 ways to the multi-channel frequency standard comparison system, of which, 1 way is connected to the reference input end of the multi-channel frequency standard comparison system, and the other 8 ways are connected to the measured input end of the multi-channel frequency standard comparison system. Since both the reference and measured signals are the same signal, then the output result of the multi-channel frequency standard comparison system is the noise floor of the measurement system itself.

Table 2. Allen standard deviation data of background noise measurement.

\begin{tabular}{llllllll}
\hline sampling time/s & CH1 & CH2 & CH3 & CH4 & CH5 & CH6 & CH7 \\
\hline $1 \mathrm{~s}$ & $2.57 \mathrm{E}-14$ & $3.98 \mathrm{E}-14$ & $4.66 \mathrm{E}-14$ & $4.72 \mathrm{E}-14$ & $3.62 \mathrm{E}-14$ & $3.47 \mathrm{E}-14$ & $4.31 \mathrm{E}-14$ \\
$10 \mathrm{~s}$ & $3.91 \mathrm{E}-15$ & $6.10 \mathrm{E}-15$ & $6.98 \mathrm{E}-15$ & $7.40 \mathrm{E}-15$ & $5.74 \mathrm{E}-15$ & $5.18 \mathrm{E}-15$ & $6.73 \mathrm{E}-15$ \\
$100 \mathrm{~s}$ & $6.39 \mathrm{E}-16$ & $9.17 \mathrm{E}-16$ & $8.23 \mathrm{E}-16$ & $9.37 \mathrm{E}-16$ & $8.08 \mathrm{E}-16$ & $7.91 \mathrm{E}-16$ & $8.38 \mathrm{E}-16$ \\
$1000 \mathrm{~s}$ & $1.36 \mathrm{E}-16$ & $3.21 \mathrm{E}-16$ & $3.68 \mathrm{E}-16$ & $3.44 \mathrm{E}-16$ & $2.49 \mathrm{E}-16$ & $2.99 \mathrm{E}-16$ & $3.25 \mathrm{E}-16$ \\
\hline
\end{tabular}

$5 \mathrm{E}-14$

\section{Allen Standard Deviationбy( $\tau)$}

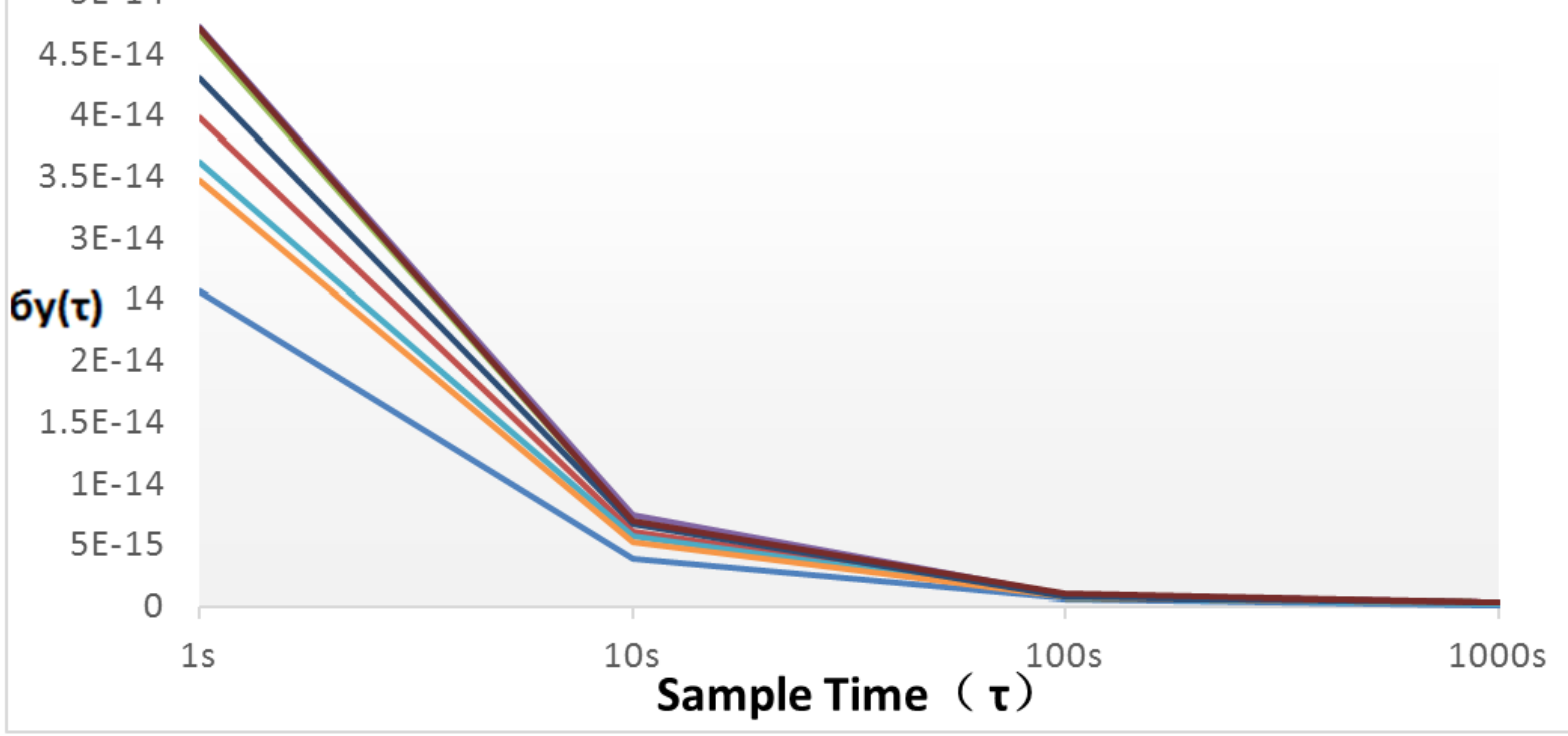

Figure 7. Allen standard deviation data of background measurement.

\subsection{Comparison and Measurement of Experimental Results}

In order to further illustrate the validity of the comparison measurement results, it is necessary to use other frequency standard measuring equipment to compare and measure the same frequency standard signals, so as to explain the measurement results of different equipment. According to the existing conditions of the laboratory, phase noise measurement system 5120A [16] produced by American Microsemi Company and phase noise measurement system
VCH314 produced by Russian Company were selected as frequency standard comparison equipment for comparison. As well as domestic equipment PO7D-2 of Shijiazhuang Shuying Instrument Co., Ltd. and PO7D-2 of $\mathrm{Xi}$ 'an Hongtai Time-Frequency Technology Co., Ltd. The measurement principle of 5120A, HT5360 is dual mixer time difference method, the measurement principle of VCH314, PO7D-2 is frequency difference multiplication method. The above equipment is measured by frequency standard 1 and frequency standard 2 respectively, and the results are shown in Table 2. 
Table 3. Comparison data of Allen standard deviation for foreign equipment.

\begin{tabular}{|c|c|c|c|}
\hline $\begin{array}{l}\text { Allen standard } \\
\text { deviation }\end{array}$ & $\begin{array}{l}\text { Multi-channel frequency standard comparison } \\
\text { measuring equipment TM-PC08 }\end{array}$ & America Microchip 5120A & Russia VCH314 \\
\hline$\sigma y(1 s)$ & $1.2 \mathrm{E}-12$ & $1.4 \mathrm{E}-12$ & $1.3 \mathrm{E}-12$ \\
\hline$\sigma y(10 s)$ & $4.0 \mathrm{E}-13$ & $3.9 \mathrm{E}-13$ & $4.3 \mathrm{E}-13$ \\
\hline$\sigma y(100 s)$ & $1.8 \mathrm{E}-13$ & $2.1 \mathrm{E}-13$ & $2.3 \mathrm{E}-13$ \\
\hline$\sigma y(1000 s)$ & $2.4 \mathrm{E}-13$ & $2.3 \mathrm{E}-13$ & $1.1 \mathrm{E}-13$ \\
\hline measuring method & Dual mixer time difference measurement & $\begin{array}{l}\text { Dual mixer time difference } \\
\text { measurement }\end{array}$ & $\begin{array}{l}\text { Frequency difference multiplication } \\
\text { method }\end{array}$ \\
\hline
\end{tabular}

Table 4. Comparison data of Allen standard deviation for domestic equipment.

\begin{tabular}{|c|c|c|c|}
\hline \multicolumn{4}{|c|}{ Comparison of equipment with domestic frequency standard } \\
\hline & $\begin{array}{l}\text { Multi-channel frequency standard } \\
\text { comparison measuring equipmentTM-PC08 }\end{array}$ & $\begin{array}{l}\text { Shijiazhuang Shuying } \\
\text { Instrument Co. Ltd PO7D-2 }\end{array}$ & $\begin{array}{l}\text { Xi 'an Hongtai Time Frequency } \\
\text { Technology Co. Ltd HT5360 }\end{array}$ \\
\hline Index comparison & $\begin{array}{l}\sigma \mathrm{y}(1 \mathrm{~s}) \leq 1.5 \mathrm{E}-13 \\
\sigma \mathrm{y}(10 \mathrm{~s}) \leq 2.0 \mathrm{E}-14 \\
\sigma \mathrm{y}(100 \mathrm{~s}) \leq 3.0 \mathrm{E}-15 \\
\sigma \mathrm{y}(1000 \mathrm{~s}) \leq 5.0 \mathrm{E}-16\end{array}$ & $\begin{array}{l}\sigma \mathrm{y}(1 \mathrm{~s}) \leq 1.0 \mathrm{E}-12 \\
\sigma \mathrm{y}(10 \mathrm{~s}) \leq 2.0 \mathrm{E}-13 \\
\sigma \mathrm{y}(100 \mathrm{~s}) \leq 3.0 \mathrm{E}-14\end{array}$ & $\begin{array}{l}\leq 5 \mathrm{E}-13 / 1 \mathrm{~s} \text { (The standard version) } \\
\leq 8 \mathrm{E}-14 / 1 \mathrm{~s} \text { (The high-end version) } \\
\text { The sampling time of Allen variance ranges } \\
\text { from } 1 \mathrm{~ms} \text { to } 100000 \mathrm{~s}\end{array}$ \\
\hline Measuring project & $\begin{array}{l}\text { Long and short term stability, Daily frequency } \\
\text { drift, Daily fluctuation, daily ageing, } \\
\text { Frequency accuracy }\end{array}$ & $\begin{array}{l}\text { Short-term frequency stability, } \\
\text { Daily fluctuation, daily ageing, } \\
\text { Frequency accuracy }\end{array}$ & Long and short term stability \\
\hline measuring method & Dual mixer time difference measurement & $\begin{array}{l}\text { Frequency difference } \\
\text { multiplication method }\end{array}$ & Dual mixer time difference measurement \\
\hline
\end{tabular}

From the data in Tables 3 and 4 above, it can be concluded that the overall measurement results of TM-PC08 are relatively close to those of 5120A and VCH314, and the Allen standard deviation of the two at the sampling time point is less different. The two devices can reflect the comparison results of frequency standard 1 and frequency standard 2 without distortion. Shijiazhuang Shuying Instrument Co., Ltd. PO7D-2 background index and two domestic equipment Allen standard deviation background index is an order of magnitude higher. PO7D-2 cannot measure the long-term stability and daily frequency stability of rubidium atom frequency standard. The multi-channel frequency standard comparator TM-PC08 can realize the dual functions of PO7D-2 and multi-channel intelligent phase comparator. It can realize automatic measurement of cesium atom frequency standard, rubidium atom frequency standard, high stable crystal oscillator, quartz crystal oscillator, frequency counter and other frequency characteristics (frequency stability, frequency drift rate, frequency aging rate, frequency daily fluctuation, frequency accuracy) with $10 \mathrm{MHz}$ time-base output.

\section{Conclusion}

Developed a measuring equipment for multi-channel frequency standard comparison, it has been running for more than 2 years in time keeping laboratory, National Institute of Metrology. The system is stable and reliable, its advantage is that the data of frequency deviation, stability (Allen deviation), daily fluctuation, daily aging rate, frequency drift rate and so on can be calculated simultaneously for 8 channels. The software function is rich, the man-machine interaction is intuitive and simple. The system has good expansion performance and can be customized to measure the number of channels flexibly according to user needs. Stability index is better than: 2E-14 (1s); 5E-15 (10S); 1.5E-15 (1000s); $5.0 \mathrm{E}-16(1000 \mathrm{~s})$, it has carried out the value transfer of frequency standard measurement in National Institute of Metrology to verify and calibrate the long and short term frequency characteristic indexes of various atomic frequency standards and quartz crystal frequency standards, so as to ensure the accuracy and reliability of time and frequency values in China. Liaoning Institute of Metrology, Henan Institute of Metrology, Guangdong Institute of Metrology, Guizhou Institute of Metrology, 92957 Army and Huizhongxingzhi Technology (Beijing) Co., Ltd have also purchased this equipment.

\section{References}

[1] Zhang A M, Gao X X, Ning D Y, et al. Realization of 8-channel DMTD Measurement System [J]. Acta Metrologica Sinica, 2009, 30 (6): 563-566.

[2] Zhao K J, Zhang A M, Xu Q H, et al. Experiment research on digital DMTD measurement [J]. Chinese Journal of Scientific Instrument, 2014, 35 (12): 2858-2865.

[3] Sojdr L, Cermak J, Barilet R. Optimazation of Dual - maxier Time - Difference multiplier [A]. Proceedings of the 18th European Frequency and Time Forum (EFTF) [C], Guildford, UK, 2004.

[4] 5120A, 5120A-01, and 5115A Phase Noise Test Set Operations and Maintenance Manual [M], Microchip, Inc, USA, 2019.

[5] 3120A, Phase Noise Test Probe User Guide [M], Microchip, Inc, USA, 2019.

[6] VCH-315, VCH-314 Frequency Comparator Operation Manual [M], "VREMYA-CH”, Inc, Russia, 2019.

[7] GREENHALL C A. Common - source phase error of a dual mixer stability analyzer [C]. TMO Progress Report, Califormia, USA : Jet Propulsion Laboratory, 2000. 
[8] GROVE J, HEIN J, MARTIN R A, et al. Comparison of heterodyne and direct-sampling techiques for phase- difference measurements [C]. NCSL International Workshop and Symposium, 2005.

[9] JJG 292-2009 Verification Regulation of Rubidium Atomic Frequency Standard [S].

[10] JJG 492-2009 Verification Regulation of Frequency Standard of Cesium Atom [S].

[11] JJG 1004-2005 Verification Regulation of Hydrogen Atom Frequency Standard [S].

[12] JJG 545-2015 Verification Regulation of Frequency Comparator [S].

[13] JJG 181-2005 Verification Regulation of Frequency Standard of Quartz Crystal [S].

[14] JJG 349-2014 Verification Regulation of Universal Counters [S].

[15] JJG 841-2012 Verification Regulation of Microwave Frequency Ccunter $[\mathrm{S}]$.

[16] GROVE J, HEIN J, RETTA R J, et al. Direct- digital phase -noise measurement [C]. 2004 IEEE International Ultrasonics, Ferroelectrics, and Frequency Control Joint 50th Anniversary Conference, 2004: 287-291.

\section{Biography}

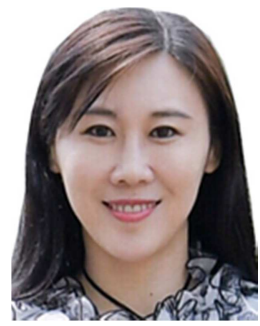

Yue Zhang received M.S. degree from National Institute of Metrology (NIM) in 2003. She is an associate research fellow in National Institute of Metrology (NIM), China, since then she has been working in Time and frequency Division of NIM. Her research interests concern: frequency standard, spectrum analyzer measurement, phase noise measurement.

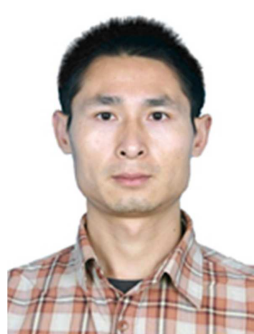

Yangqi Liu received a M.S. degree from Beijing University of Aeronautics and Astronautics in 2006. He is a senior engineer of Xinghan Technology (Beijing) Co., Ltd., he has been working in time and frequency technology research. His research interests: atomic time, time measurement, frequency measurement, satellite time service.

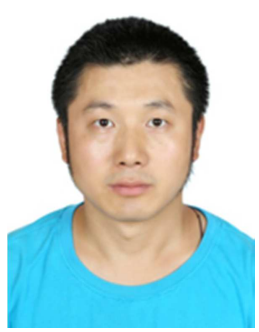

Tao Lin was born in Beijing, China in 1988. He graduated from Beijing Petroleum and Chemical Technology University with a bachelor's degree in 2010. He is an assistant engineer in National Institute of Metrology, China. He research interests concern: frequency Standard measurement, spectrum analyzer measurement.

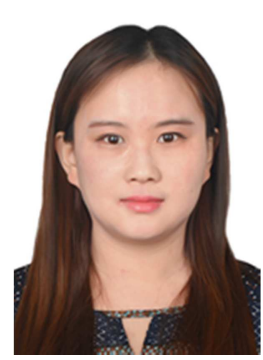

Xiaodi Yuan was born in Beijing, China in 1990. She graduated from Beijing Union University (China) with a bachelor's degree in 2012. She is an assistant engineer in National Institute of Metrology, China. Her research interests concern: frequency standard measurement.

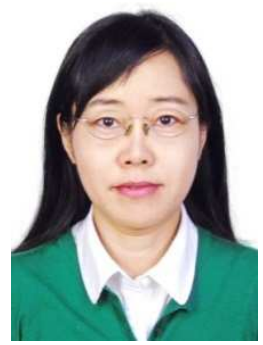

Aimin Zhang received B.S. degree from Peking University in 1987, M.S. degree from National Institute of Metrology (NIM) in 1990, since then she has been working in Time and frequency Division of NIM. She is in charge of generation and maintenance of UTC (NIM) which is the time and frequency primary standard in china. Currently she is APMP TCTF chair.

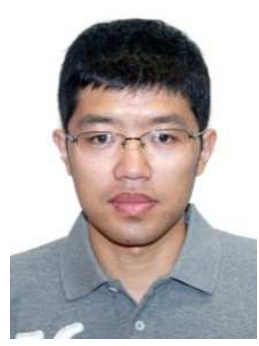

Yuzhuo Wang received the Ph.D. degree from Beijing Institute of Technology, Beijing, China, in 2015. He is currently in the Division of Time and Frequency, National Institute of Metrology (NIM), China. His current research interests include atomic time scale algorithm and precise measurement about time and frequency. 